\title{
Millimeter-Wave Beam Scattering by Field-Aligned Blobs in Simple Magnetized Toroidal Plasmas
}

\author{
O. Chellaï, ${ }^{1, *}$ S. Alberti, ${ }^{1}$ M. Baquero-Ruiz, ${ }^{1}$ I. Furno, ${ }^{1}$ T. Goodman, ${ }^{1}$ F. Manke, ${ }^{1}$ G. Plyushchev, ${ }^{1}$ L. Guidi, ${ }^{2}$ \\ A. Koehn, ${ }^{2,3}$ O. Maj, ${ }^{2}$ E. Poli, ${ }^{2}$ K. Hizanidis, ${ }^{4}$ L. Figini, ${ }^{5}$ and D. Ricci ${ }^{5}$ \\ ${ }^{1}$ Swiss Plasma Center (SPC), École Polytechnique Fédérale de Lausanne (EPFL), 1015 Lausanne, Switzerland \\ ${ }^{2}$ Max Planck Institute for Plasma Physics, 85748 Garching, Germany \\ ${ }^{3}$ Institute of Interfacial Process Engineering and Plasma Technology, University of Stuttgart, 70174 Stuttgart, Germany \\ ${ }^{4}$ National Technical University of Athens, 10682 Athens, Greece \\ ${ }^{5}$ Istituto di Fisica del Plasma, Consiglio Nazionale delle Ricerche, 20125 Milan, Italy
}

(Received 14 November 2017; published 8 March 2018)

\begin{abstract}
The first direct experimental measurements of the scattering of a millimeter-wave beam by plasma blobs in a simple magnetized torus are reported. The wavelength of the beam is comparable to the characteristic size of the blob. In situ Langmuir probe measurements show that fluctuations of the electron density induce correlated fluctuations of the transmitted power. A first-principles full-wave model, using conditionally sampled 2D electron density profiles, predicts fluctuations of the millimeter-wave power that are in agreement with experiments.
\end{abstract}

DOI: 10.1103/PhysRevLett.120.105001

The propagation of electromagnetic (EM) waves in turbulent plasmas is of fundamental importance in nature and in laboratories. EM wave scattering by plasma turbulence in the interstellar medium [1] and in the ionosphere $[2,3]$ must be modeled to correctly interpret the EM emission from celestial bodies. In magnetically confined plasmas, EM waves serve a dual purpose: First, they have a wide application in plasma diagnostics. EM waves emitted in the electron cyclotron (EC) frequency range from the confined region are used to measure plasma parameters [4]. Low-power EM waves in the infrared $[5,6]$ and in the millimeter-wave ( $\mathrm{mmw}$ ) range [7-9] are employed to probe plasma turbulence [10] and plasma waves. Second, externally injected EC beams in fusion devices [11,12] are employed for plasma heating, to drive plasma current and to stabilize neoclassical tearing modes to avoid core confinement degradation and plasma disruptions [13,14]. In all fusion devices, field-aligned coherent structures generated from the underlying turbulence, referred to as blobs, are present near the plasma edge and are characterized by a local enhancement of the electron density (up to $100 \%$ compared to the surrounding environment) [15]. By locally modifying the plasma permittivity, blobs can scatter incoming mmw's. This leads to difficulties in the interpretation of diagnostic signals [16] and to a potential loss of efficiency in EC heating and mode stabilization, as

Published by the American Physical Society under the terms of the Creative Commons Attribution 4.0 International license. Further distribution of this work must maintain attribution to the author(s) and the published article's title, journal citation, and DOI. suggested by numerical [16,17] and analytical [18] studies. Recently, concerns over the possible broadening effect of blobs on EC beams were raised for ITER $[19,20]$. The precise amount of EC-beam broadening may strongly depend on the characteristics of the turbulent structures [21] and could reach more than a factor of 2 [17], complicating the tearing mode stabilization in ITER $[19,22]$ with the present EC plant design. Experimental measurements of $\mathrm{mmw}$ scattering by blobs in fusion plasmas are hampered by diagnostic accessibility and have been, to date, limited to attempts to compare indirectly inferred heating power deposition profiles with predictions of the ray-tracing code TORAY-GA [23]. Direct experimental evidence of scattering - and the induced broadening, which is of fundamental importance for the validation of codes and quantitative predictions-is still missing.

In this Letter, we report on the first direct measurements of millimeter-wave scattering by intermittent blobs in a simple magnetized torus (SMT). The experiments are carried out in the toroidal basic-plasma-physics device named TORPEX [24]. An antenna launches a mmw beam from the top of TORPEX through a plasma featuring blobs with statistical properties similar to those observed in tokamaks [25]. A receiving antenna measures the mmwbeam power arriving at the bottom of the vessel. Using in situ two-dimensional Langmuir probe data, we show that fluctuations of the detected $\mathrm{mmw}$ power are caused by fluctuations in the plasma density associated with blobs. A first-principles full-wave numerical model predicts that blobs will scatter the mmw beam by locally modifying the plasma permittivity, at a fluctuation level that is in agreement with the experiments. 


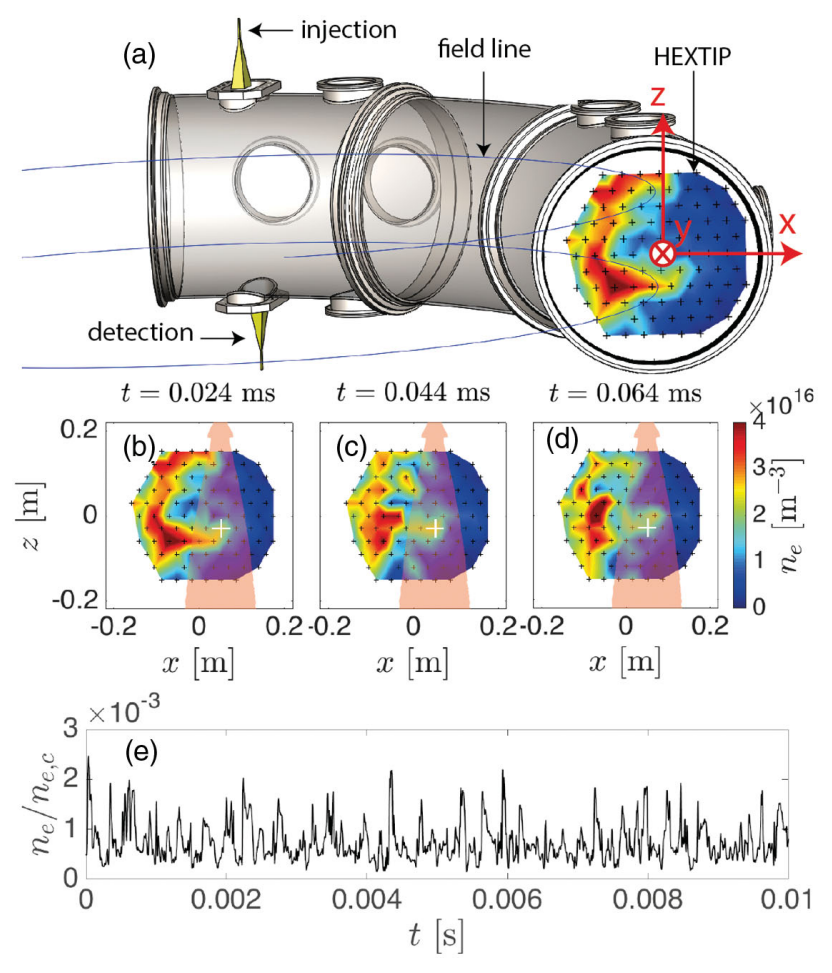

FIG. 1. TORPEX illustration and raw data. (a) Illustration of the experimental setup in TORPEX with a helical magnetic field line (in blue) in a simple magnetized torus configuration. A mmw beam is injected in $X$ mode from the top of the vessel and detected at the bottom through dielectric windows. In situ 2D measurements of density fluctuations associated with blobs are obtained from the HEXTIP array of 95 Langmuir probes at the positions indicated in black. (b)-(d) Snapshots of density fluctuations showing (b) blob formation, (c) detachment, and (d) radial propagation (in the vicinity of the white cross). The shaded area represents the mmw beam at the full width at half maximum of the mmw power from COMSOL simulations in vacuum. (e) Time evolution of the electron density normalized to the $X$-mode cutoff electron density as measured at the location in white in (b).

In TORPEX (major radius $R=1 \mathrm{~m}$, minor radius $r=0.2 \mathrm{~m}$ ), a SMT configuration is produced by imposing a vertical magnetic field $B_{z}=2.1 \mathrm{mT}$ on a toroidal magnetic field $B_{y}=74 \mathrm{mT}$. This results in helical field lines terminating on the bottom and the top of the ring-shaped vacuum vessel, as shown in Fig. 1(a). Hydrogen plasmas are generated by injecting $300 \mathrm{~W}$ of microwave power at $2.45 \mathrm{GHz}$. Consistent with previous detailed comparisons of numerical simulations and experimental measurements [26], the plasma is dominated by an ideal interchange wave localized around $x \approx-5 \mathrm{~cm}$, with parallel and perpendicular wave numbers $k_{\|} \approx 0$ and $k_{z}=2 \pi / \Delta \approx 35 \mathrm{~m}^{-1}$, respectively. Here, $\Delta=2 \pi R B_{z} / B_{y} \approx 17.8 \mathrm{~cm}$ is the vertical displacement of a field line after one toroidal turn. The ideal interchange wave is associated with coherent fluctuations of the electron density at a dominant frequency of $\sim 4.15 \mathrm{kHz}$. The spectral power density of the fluctuations at this frequency is maximal at $x \approx-5 \mathrm{~cm}$ and decreases with $x$, falling to $\approx 10 \%$ of its peak value at $x=5 \mathrm{~cm}$. The region defined by $x \gtrsim-5 \mathrm{~cm}$ is characterized by the presence of blobs, which are intermittently generated from the ideal interchange wave [27] and propagate radially outwards, i.e., in the direction of increasing $x$ values, into the low-field side region of TORPEX [28]. More details on similar scenarios are given in Refs. [28-32].

A blob example is shown in Figs. 1(b)-1(d), where 2D measurements of the electron density $n_{e}$ are computed from ion-saturation current data obtained using the hexagonal turbulence imaging probe (HEXTIP) diagnostic [33] in combination with triple probe electron temperature measurements [34]. The evaluated density is cross-checked with the experimentally determined location of the upperhybrid resonance [34]. A typical blob has a half-widthhalf-maximum (HWHM) size of $a \sim 2.5 \mathrm{~cm}$ for density fluctuations $\delta n_{e} / n_{e}$ up to $100 \%$ and propagates radially at about $v_{x} \approx 10^{3} \mathrm{~m} / \mathrm{s}$ [28]. Here, density fluctuations are defined as $\delta n_{e}=n_{e}-\left\langle n_{e}\right\rangle_{t}$, where $\left\langle n_{e}\right\rangle_{t}$ is the timeaveraged density. The time evolution of $n_{e}$ shown in Fig. 1(e) is obtained in the TORPEX blob region using the probe represented by the white cross [Fig. 1(b)-1(d)]. $n_{e}$ is normalized to $n_{e, c}=1.01 \times 10^{19} \mathrm{~m}^{-3}$, the cutoff density of the extraordinary mode (the $X$ mode) of the mmwinjection frequency [12] (see below). Here, $n_{e} / n_{e, c} \sim 10^{-3}$ and, hence, the probing frequency is far from the cutoff. Rapid increases in $n_{e}$ are associated with blobs passing the probe.

For the present experiments, we have developed a dedicated mmw-injection and -detection system. The system is located $90^{\circ}$ away in the toroidal direction from HEXTIP, as shown, schematically, in Fig. 1(a). A lowpower $(7 \mathrm{~mW}) \mathrm{mmw}$ beam at $f=29.7 \mathrm{GHz}$ (wavelength, $\lambda \approx 10.1 \mathrm{~mm}$ in vacuum) is launched in the $X$ mode from the top of the device through a Plexiglas window using a pyramidal horn antenna at $x=5 \mathrm{~cm}, z=23 \mathrm{~cm}$. The $X$ mode component of the mmw transmitted power is detected at the bottom of the device using a pyramidal horn antenna and a Schottky diode. The dimension of the horn is $4.2 \mathrm{~cm}$ in the $x$ direction and $5.5 \mathrm{~cm}$ in the $y$ direction. The signal is digitized at $250 \mathrm{kHz}$. The detector can be moved radially, to probe the mmw beam in the range $x \in[-3,12] \mathrm{cm}$ and thus reconstruct the time-averaged radial profile of the detected power, as well as its fluctuations. The resulting profile is a convolution between the radiation pattern of the detector and the transmitted mmw beam. The profile in the absence and in the presence of a plasma is shown in Fig. 2(a). Both profiles can be approximated by a Gaussian with a HWHM of $w_{0} \approx 6 \mathrm{~cm}$.

Figure 2(a) shows that the time-averaged detected profile is not strongly affected by the presence of the plasma. Figure 2(b) compares the time evolution of the power, detected at $x=5 \mathrm{~cm}$ (the beam center) and normalized to the time-averaged power, in the absence and in the presence of plasma. Figure 2(c) compares the standard deviation of these two signals at several detector positions within the 


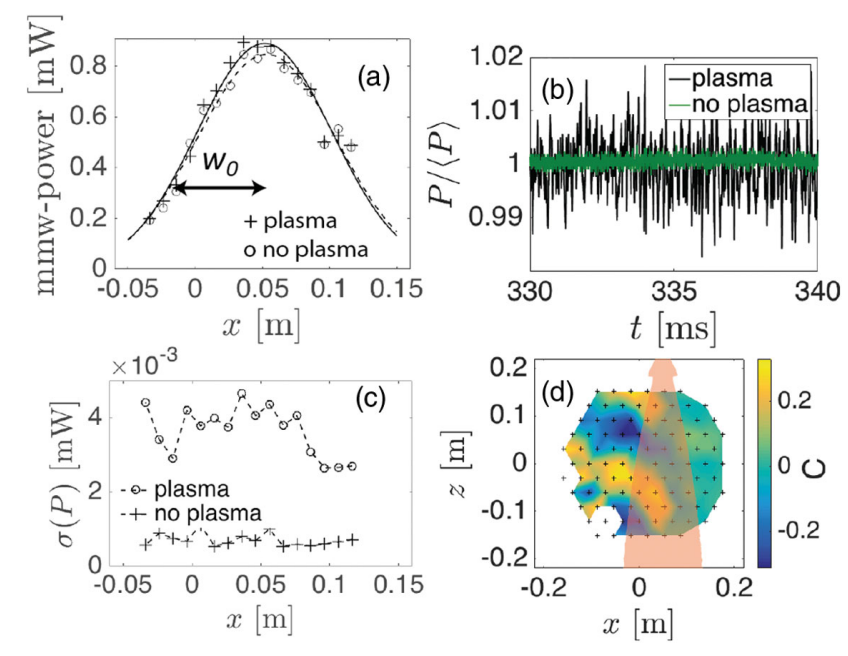

FIG. 2. Statistical properties of the detected mmw-power signal. (a) Time-averaged detected mmw power with and without plasma. (b) Comparison of the time evolution of the detected mmw power, normalized to its mean value, with (black) and without (green) plasma. (c) Standard deviation of the detected mmw-power signal. (d) Cross-correlation between the mmw power measured at $x=5 \mathrm{~cm}$ and the electron density measured at each probe of HEXTIP.

accessible $x$ range. Figures 2(b)-2(c) show that plasmas cause fluctuations $\delta P(x, t)=P(x, t)-\langle P(x, t)\rangle_{t}$ in the detected mmw-beam power that are not present in the absence of a plasma. These fluctuations are associated with an increase or a decrease of the detected $\mathrm{mmw}$ power in the entire range $x \in[-3,12] \mathrm{cm}$ [Fig. 2(c)]. Figure 2(d) shows the 2D distribution of the coefficient of the cross-correlation at zero time lag between $\delta P(x, t)$ measured with the detecting antenna at $x=5 \mathrm{~cm}$ and $\delta n_{e}(\mathbf{x}, t)$ obtained with HEXTIP [33].$\delta n_{e}(\mathbf{x}, t)$ and $\delta P(x=5 \mathrm{~cm}, t)$ are positively correlated in some regions and negatively correlated in others. When the cross-correlation is computed with a nonzero time lag between $\delta P(x, t)$ measured at $x=5 \mathrm{~cm}$ and $\delta n_{e}(\mathbf{x}, t)$, the same structures are obtained, but they are shifted vertically. When passing through the beam, blobs are still coherent with the ideal interchange wave located in the region $x \lesssim-5 \mathrm{~cm}$, which explains the finite value of the cross-correlation measured outside the beam path. Figure 2(d) thus shows that depending on the spatial location of a blob in the poloidal plane, the blob can reduce (negative correlation) or increase (positive correlation) the power detected at the location of the detection horn. This behavior is explained below with the help of full-wave simulations (see Fig. 4).

To elucidate the effect of a typical blob on the mmw beam, we perform conditional sampling (CS) [29] of the mmwpower signal and the HEXTIP signals over many blob events with a time window centered around each blob event. Events are defined as local maxima that fulfill the condition $n_{e}>\left\langle n_{e}\right\rangle_{t}+3 \sigma$, with $\sigma$ being the standard deviation of $n_{e}$ calculated over the entire time trace. All quantities
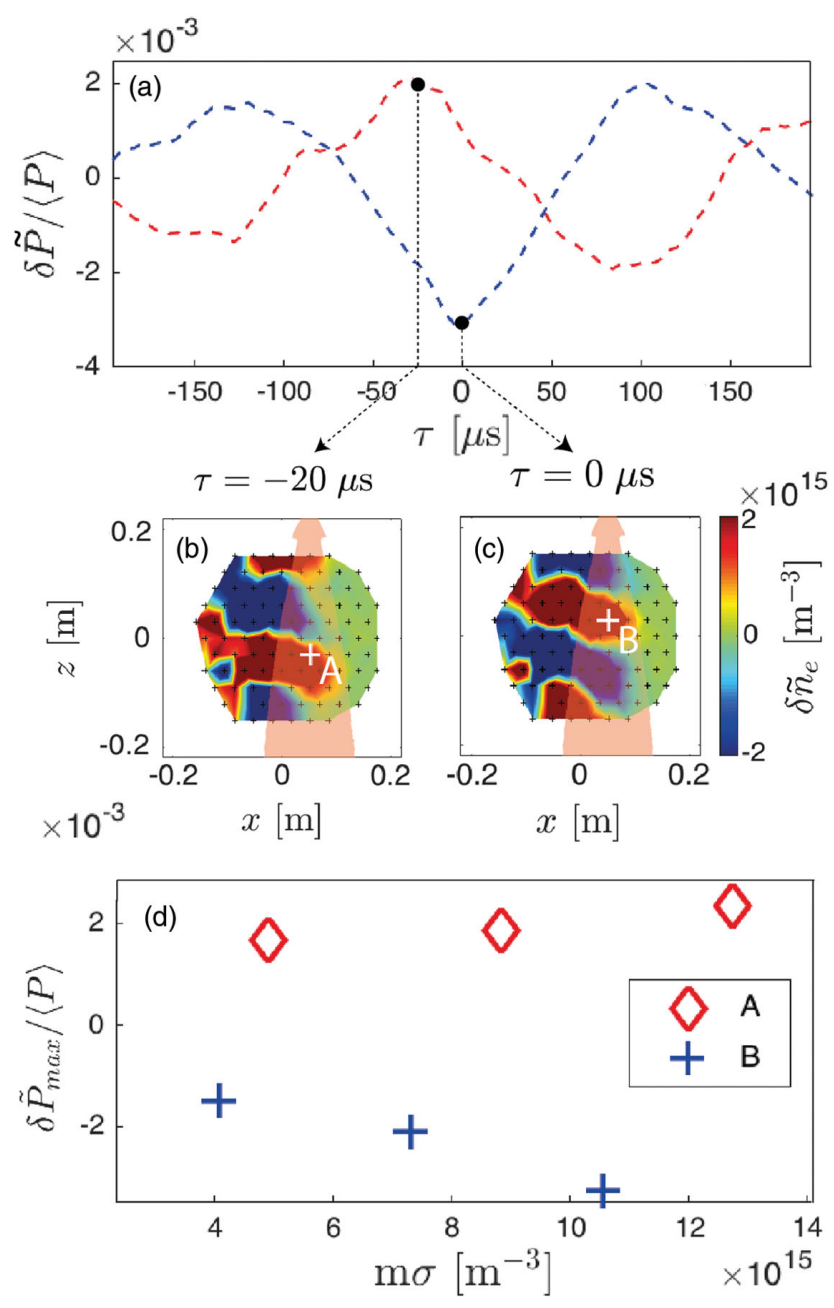

FIG. 3. Conditional sampling (CS) using two different probes to detect blobs. Note that, since $k_{\|} \approx 0$, a single blob would appear twice within the poloidal cross section of TORPEX. (a) Red (probe $A$ ) and blue (probe $B$ ) dashed lines show the result of CS using the probes identified in white in (b) and (c). (b),(c) Electron density fluctuations at $\tau=-20$ and $0 \mu$ s, respectively. (d) Maximum amplitude of fluctuations of the normalized detected power $\delta \tilde{P}_{\max } /\langle P\rangle$ as a function of the range of the CS threshold, taking probes $A$ and $B$ as references.

obtained with CS are labeled with a tilde, except $\tau$, which is the time relative to the blob event; $\tau=0 \mu$ s corresponds to the detection of the blob. Either of the two HEXTIP probesshown in white in Figs. 3(b) and 3(c)-is used for blob detection. Their location is chosen to correspond approximately to the positions of the maximum and minimum values of the cross-correlation in the blob region in Fig. 2(d). The results of CS of the detected mmw power over $N \approx 1000$ events are presented in Fig. 3(a). Errors in the averaging process are proportional to $1 / \sqrt{N}$ and are on the order of $10^{-4}$. The associated variation of power is on the order of $\sim 0.1 \%$. The results show that blobs detected at the two different vertical locations have a different effect on the locally detected mmw power. 
At $\tau=-20 \mu \mathrm{s}$, a blob passing through $A$ [Fig. 3(a)] increases the mmw power transmitted to the horn. A blob passing at $B$ decreases the detected power. The dependence of the amplitude of the two extrema in Fig. 3(a) upon the density in the range used for CS event selection is addressed in Fig. 3(d). Several classes of blobs are defined by the condition $\left\langle n_{e}\right\rangle_{t}+m \sigma<n_{e}<\left\langle n_{e}\right\rangle_{t}+(m+0.5) \sigma$, with $m=1,2,3$. The extrema occur in a time window of $+/-20 \mu$ s relative to the ones in Fig. 3(a). Figure 3(d) shows that blobs with larger density fluctuations have larger effects on the mmw-detected power; the sign of the fluctuation depends on the blob location.

Numerical simulations are used to understand the underlying scattering phenomena. The propagation of a mmw in magnetized plasmas is typically modeled in a highfrequency or short-wavelength approximation [35,36] where ray- or beam-tracing codes provide the trajectory of the mmw refracted by plasma inhomogeneities. For blobs with a typical scale $a$ such that $\lambda / a \sim 1$ (as here), this approximation is no longer valid, and a more recent generalization of this approach [21] or a full-wave model is needed to capture the underlying physics [18]. We use 2D full-wave numerical simulations based on COMSOL MULTIPHYSICS $[37,38]$. Since the beam angular frequency $\omega$ satisfies $\omega \gg\left|\partial_{t} n_{e} / n_{e}\right| \equiv T^{-1}$, one can show, by means of a two-scale expansion, that for $t$ in a time interval $[\tau, \tau+\Delta \tau], \Delta \tau \sim T \gg 2 \pi / \omega$, the beam is well approximated by the solution of the cold plasma model with electron density frozen at a considered time $\tau$. Within this "frozen-blob" approximation, we can regard the plasma as stationary and thus reduce the cold plasma model to a single equation for the electric field $\mathbf{E}(\mathbf{x}, \omega)$ in the frequency domain, namely,

$\boldsymbol{\nabla} \times[\boldsymbol{\nabla} \times \mathbf{E}(\mathbf{x}, \omega)]+\frac{\omega^{2}}{c^{2}} \hat{\epsilon}_{r}(\mathbf{x}, \omega) \mathbf{E}(\mathbf{x}, \omega)=\mathbf{S}(\mathbf{x}, \omega)$,

where $c$ is the speed of light in free space, and $\hat{\epsilon}_{r}(\mathbf{x}, \omega)$ is the cold plasma dielectric tensor [4] computed with the equilibrium magnetic field and with density $n_{e}(\mathbf{x}, \tau)$ frozen at time $\tau$. The source $\mathbf{S}$ accounts for the $T E_{10}$ mode of the antenna, with the polarization chosen in order to excite the $X$ mode. Equation (1) is solved for a sequence of points in time $\tau_{i}$ with $\Delta \tau=\tau_{i+1}-\tau_{i}=4 \mu \mathrm{s} \gg 2 \pi / \omega$, thus obtaining a sequence of beams $\mathbf{E}_{i}(\mathbf{x}, \omega)$ corresponding to different density snapshots. The time-resolved $2 \mathrm{D}$ profiles of the electron density $\tilde{n}_{e}\left(\mathbf{x}, \tau_{i}\right)$ are calculated by applying the CS procedure to the experimental density measurements [Figs. 4(a) and 4(b); the contours] and are used to compute $\hat{\epsilon}_{r}$. Given that blobs are field-aligned structures with $k_{\|} \approx 0$ and that magnetic field lines are helical, the 2D electron density profile at the toroidal location of the mmw system is similar to the one measured by HEXTIP (toroidally displaced by $90^{\circ}$ from the mmw system), but shifted vertically by $\Delta / 4 \approx 4.5 \mathrm{~cm}$. In practice, we compute the $2 \mathrm{D}$ electron density profile using, as a reference, two probes that are

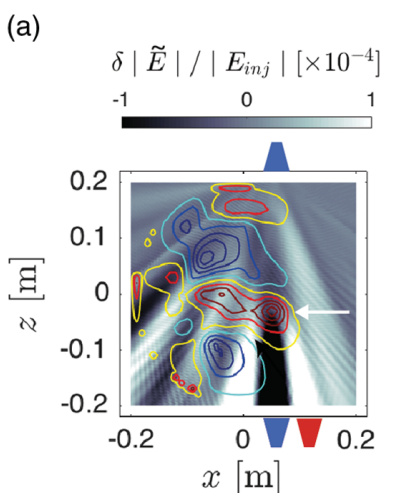

(b)
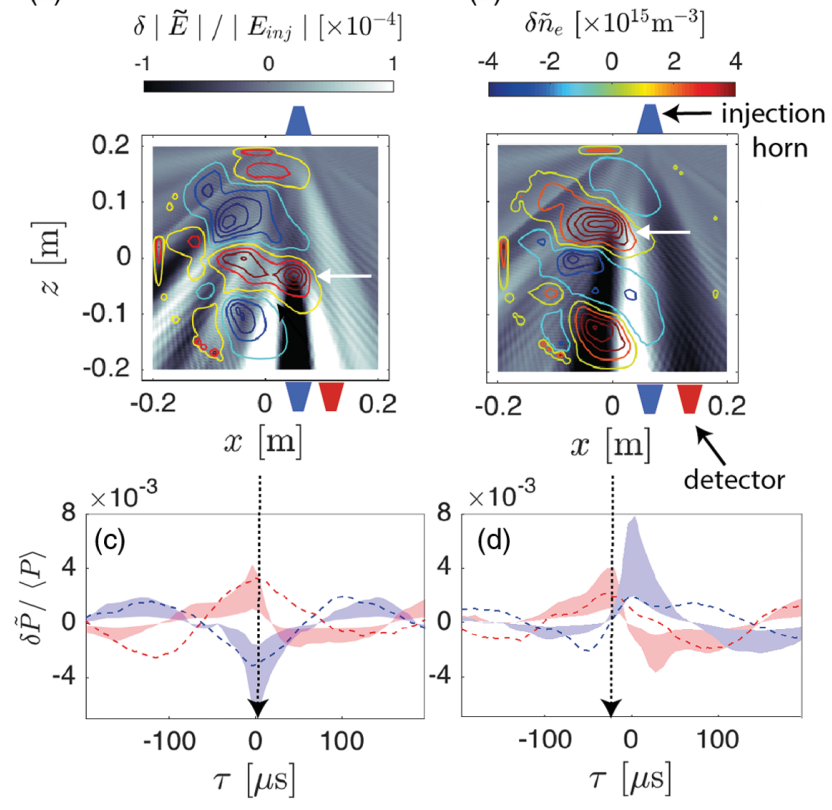

FIG. 4. Full-wave simulation results. (a), (b) Fluctuations of the electric field amplitude associated with the blobs identified in Figs. 3(b) and 3(c) projected to the poloidal plane of the mmw system. The detected electric field is normalized to the electric field maximum of the launching horn $\left|E_{\text {inj }}\right|$. (c), (d) Comparison of the time evolution of the mmw power at the two positions of detection identified by the blue and red horns in (a) and (b). The white arrows indicate a blob. The CS time evolution of the detected mmw power is obtained using probes $A$ and $B$ from Figs. 3(b) and 3(c) to detect blobs. Experimental results (the dashed lines) are compared to numerical simulations (the shaded areas).

vertically distant [i.e., from $A$ and $B$; Figs. 3(c) and 3(d)] by $\Delta / 4$. Absorbing boundary conditions [37] were used at the edge of the computational domain and give numerical results in agreement with the experiments. Absorbing boundary conditions are thus used in the following. Perfectly reflecting boundary conditions were also used on a larger domain approximating the vacuum chamber cross section, but they did not provide satisfactory results. One reasonable explanation is that mmw's reflecting off of metallic surfaces can, in the real toroidal three-dimensional case, propagate away from the region near the beam and thus dissipate the mmw power.

Numerical simulations are compared to experiments in Figs. 4(c) and 4(d). Consistent with the expected systematic uncertainties in $n_{e}$ calculated from ion-saturation data $[39,40]$, we estimate uncertainties of the model by bounding the outcome with results obtained using $0.5 n_{e}$ and $2 n_{e}$. The shaded areas in Figs. 4(c) and 4(d) indicate this variability, which shows good agreement between the numerical simulations and the experiments. The agreement can be quantified by computing the coefficient of the cross-correlation between the numerically obtained and experimentally measured $\delta \tilde{P}(x, t)$ values at zero time lag. We find that 
the coefficient ranges between 0.45 and 0.90 for the red and blue curves, respectively, in Fig. 4(c). Figures 4(a) and 4(b) show the fluctuation $\delta\left|\tilde{\mathbf{E}}_{i}(\mathbf{x}, \omega)\right|$ of the electric field of the mmw beam associated with the two configurations of electron densities described above. In the two cases presented here, a blob defracts the beam as detailed in Refs. [38,41,42]. In Fig. 4(a), the blob is at the center of the mmw beam (i.e., $x=5 \mathrm{~cm}$ ). Defocusing of the mmw beam occurs in the wake of the blob and causes a decrease of the detected power at the beam center [the blue lines in Fig. 4(c)]. The power is spread out, which results in an increase in power density and transmission at the beam edges, as shown in red in Fig. 4(c). Figure 4(b) presents another situation where the blob is located at the beam edge. Only part of the beam is defocused. The beam power at the edge is refracted to the center, resulting in an increase of the power detected at the beam center, as shown in blue in Fig. 4(d). Here, the structure with negative fluctuations of the electron density also contributes to the partial focusing of the beam.

In summary, we presented in this Letter the first experimental results of mmw-beam scattering by blobs of size $a$ comparable to the beam wavelength $\lambda$ in a simple magnetized toroidal plasma. To our knowledge, and compared to similar previous experiments [7-9], this is the first successful attempt to understand mmw scattering using in situ electron density measurements. Combining these measurements with first-principles full-wave numerical simulations, we showed that electron density fluctuations associated with plasma blobs, with $\delta n_{e}$ values as small as $\sim 10^{-3} n_{e, c}$, defocus the mmw beam in the wake of the blob structures, resulting in mmw-power fluctuations that increase monotonically with the blob amplitude. These results represent an important test case for validating numerical simulations and the theory of millimeter-wave propagation in turbulent plasmas-particularly for predicting EC-beam broadening in ITER, where the $\lambda / a$ ratio is expected to be similar to the one in this Letter, according to blob stability estimates [15]. In ITER, density fluctuations, predicted at the level $\delta n_{e} /$ $n_{e, c} \sim 1 \%$ [21], would further increase the scattering effect. Finally, we established a reliable methodology bringing together experimental data and numerical simulations which will be applied to fusion plasmas, starting with the Tokamak à Configuration Variable (TCV) tokamak, where EC-beam scattering investigations have recently begun [43].

This work was carried out within the framework of the EUROfusion Consortium and received funding from the Euratom Research and Training Programme under Grant Agreement No. 633053. The views and opinions expressed herein do not necessarily reflect those of the European Commission. This work was supported in part by the Swiss National Science Foundation.

*oulfa.chellai@epfl.ch

[1] W. Sieber, Astron. Astrophys. 113, 311 (1982).
[2] S. Priyadarshi, Surv. Geophys. 36, 295 (2015).

[3] F. Villars and V. F. Weisskopf, Phys. Rev. 94, 232 (1954).

[4] M. Bornatici, R. Cano, O. De Barbieri, and F. Engelmann, Nucl. Fusion 23, 1153 (1983).

[5] C. M. Surko and R. E. Slusher, Phys. Rev. Lett. 37, 1747 (1976).

[6] C. M. Surko, R. E. Slusher, J. J. Schuss, R. R. Parker, I. H. Hutchinson, D. Overskei, and L. S. Scaturro, Phys. Rev. Lett. 43, 1016 (1979).

[7] V. Arunasalam and J. Sinnis, Phys. Rev. Lett. 23, 635 (1969).

[8] R. Cano, I. Fidone, J. Mendonca, and B. Zanfagna, Phys. Fluids 19, 1561 (1976).

[9] R. Rohatgi, K.-I. Chen, G. Bekefi, P. Bonoli, S. Luckhardt, M. Mayberry, M. Porkolab, and J. Villasenor, Phys. Fluids B 3, 2101 (1991).

[10] V. F. Shevchenko, R. G. L. Vann, S. J. Freethy, and B. K. Huang, J. Instrum. 7, P10016 (2012).

[11] S. Alberti, Nat. Phys. 3, 376 (2007).

[12] R. Prater, Phys. Plasmas 11, 2349 (2004).

[13] G. Gantenbein, H. Zohm, G. Giruzzi, S. Günter, F. Leuterer, M. Maraschek, J. Meskat, Q. Yu (the ASDEX Upgrade Team, and the ECRH-Group (AUG)), Phys. Rev. Lett. 85, 1242 (2000).

[14] I. G. J. Classen, E. Westerhof, C. W. Domier, A. J. H. Donné, R. J. E. Jaspers, N. C. Luhmann, Jr., H. K. Park, M. J. van de Pol, G. W. Spakman, and M. W. Jakubowski (for the TEXTOR Team), Phys. Rev. Lett. 98, 035001 (2007).

[15] S. Krasheninnikov, D. D'ippolito, and J. Myra, J. Plasma Phys. 74, 679 (2008).

[16] A. Köhn, E. Holzhauer, J. Leddy, M. B. Thomas, and R. G. Vann, Plasma Phys. Controlled Fusion 58, 105008 (2016).

[17] C. Tsironis, A. G. Peeters, H. Isliker, D. Strintzi, I. Chatziantonaki, and L. Vlahos, Phys. Plasmas 16, 112510 (2009).

[18] A. K. Ram, K. Hizanidis, and Y. Kominis, Phys. Plasmas 20, 056110 (2013).

[19] E. Poli et al., Nucl. Fusion 55, 013023 (2015).

[20] E. V. Sysoeva, F. da Silva, E. Z. Gusakov, S. Heuraux, and A. Y. Popov, Nucl. Fusion 55, 033016 (2015).

[21] A. Snicker, E. Poli, O. Maj, L. Guidi, A. Koehn, H. Weber, G. D. Conway, M. A. Henderson, and G. Saibene, Nucl. Fusion 58, 016002 (2018).

[22] Y. Peysson, J. Decker, L. Morini, and S. Coda, Plasma Phys. Controlled Fusion 53, 124028 (2011).

[23] M. W. Brookman, M. E. Austin, K. W. Gentle, C. C. Petty, D. E. Ernst, Y. Peysson, J. Decker, and K. Barada, EPJ Web Conf. 147, 03001 (2017).

[24] I. Furno et al., J. Plasma Phys. 81, 345810301 (2015).

[25] B. Labit, I. Furno, A. Fasoli, A. Diallo, S. H. Müller, G. Plyushchev, M. Podestà, and F. M. Poli, Phys. Rev. Lett. 98, 255002 (2007).

[26] P. Ricci, C. Theiler, A. Fasoli, I. Furno, B. Labit, S. H. Müller, M. Podesta, and F. M. Poli, Phys. Plasmas 16, 055703 (2009).

[27] I. Furno et al., Phys. Rev. Lett. 100, 055004 (2008).

[28] C. Theiler, I. Furno, P. Ricci, A. Fasoli, B. Labit, S. H. Müller, and G. Plyushchev, Phys. Rev. Lett. 103, 065001 (2009). 
[29] I. Furno et al., Phys. Plasmas 15, 055903 (2008).

[30] M. Podesta, A. Fasoli, B. Labit, I. Furno, P. Ricci, F. M. Poli, A. Diallo, S. H. Müller, and C. Theiler, Phys. Rev. Lett. 101, 045001 (2008).

[31] I. Furno, M. Spolaore, C. Theiler, N. Vianello, R. Cavazzana, and A. Fasoli, Phys. Rev. Lett. 106, 245001 (2011).

[32] C. Theiler, I. Furno, J. Loizu, and A. Fasoli, Phys. Rev. Lett. 108, 065005 (2012).

[33] M. Baquero-Ruiz, F. Avino, O. Chellai, A. Fasoli, I. Furno, R. Jacquier, F. Manke, and S. Patrick, Rev. Sci. Instrum. 87, 113504 (2016).

[34] C. Theiler, I. Furno, A. Kuenlin, P. Marmillod, and A. Fasoli, Rev. Sci. Instrum. 82, 013504 (2011).

[35] R. Prater et al., Nucl. Fusion 48, 035006 (2008).

[36] D. Farina, Fusion Sci. Technol. 52, 154 (2007).
[37] COMSOL, Inc., COMSOL MULTIPHYSICS RF Module User's Guide, Version 5.2a (COMSOL, Inc., Burlington, MA, 2016), https://www.comsol.com/release/5.2a.

[38] Z. C. Ioannidis, A. K. Ram, K. Hizanidis, and I. G. Tigelis, Phys. Plasmas 24, 102115 (2017).

[39] P. M. Bellan, Fundamentals of Plasma Physics (Cambridge University Press, Cambridge, England, 2008).

[40] F. F. Chen, J. D. Evans, and W. Zawalski, Plasma Sources Sci. Technol. 21, 055002 (2012).

[41] A. K. Ram and K. Hizanidis, Phys. Plasmas 23, 022504 (2016).

[42] Z. C. Ioannidis, A. K. Ram, K. Hizanidis, and I. G. Tigelis, Phys. Plasmas 24, 102115 (2017).

[43] O. Chellai, S. Alberti, I. Furno, T. Goodman, A. Koehn, L. Figini, D. Ricci, K. Hizanidis, P. Papagiannis, C. Tsironis, and (the TCV Team), EPJ Web Conf. 157, 03008 (2017). 